Psychotherapeut 2021 66:209-216 https://doi.org/10.1007/s00278-021-00501-w Angenommen: 11. Februar 2021 Online publiziert: 18. März 2021

(c) Der/die Autor(en) 2021

Vera Clemens ${ }^{1}$. Franziska Köhler-Dauner ${ }^{1}$ Ferdinand Keller ${ }^{1}$ Ute Ziegenhain ${ }^{1}$ Jörg M. Fegert' ${ }^{1}$ Michael Kölch ${ }^{2}$

${ }^{1}$ Klinik für Kinder- und Jugendpsychiatrie/Psychotherapie, Universitätsklinikum Ulm, Ulm, Deutschland ${ }^{2}$ Klinik für Psychiatrie, Neurologie, Psychosomatik und Psychotherapie im Kindes- und Jugendalter, Universitätsmedizin Rostock, Rostock, Deutschland

\title{
Gewalt in intimen Partnerschaften und psychische Probleme bei Kindern und Jugendlichen
}

\section{Online-Survey während der COVID-19- Pandemie}

\begin{abstract}
Belastende Kindheitserlebnisse (,, adverse childhood experiences", ACE) erhöhen das Risiko für Gewalt in intimen Partnerschaften und diese wiederum das Risiko für psychische Probleme der eigenen Kinder. Es besteht die berechtigte Sorge, dass sich ein vorliegendes Risiko für das Erleben von häuslicher Gewalt in Familien aufgrund der verschlechterten Lebensbedingungen im Rahmen der durch die "coronavirus disease 2019“' (COVID-19) ausgelösten Pandemie verwirklicht bzw. sich bereits ausgelebte Gewalt verstärkt. Die vorliegende Untersuchung ist gemäß dem Wissen der Autoren eine der ersten, die sich dieses Themas unmittelbar während der Lockdown-Maßnahmen in Deutschland angenommen hat.
\end{abstract}

\section{Hintergrund und Fragestellung}

Unter häuslicher Gewalt wird körperliche, aber auch psychische, sexuelle und wirtschaftliche Gewalt im Nahraum eines Haushalts verstanden, englisch als "domestic violence“ oder auch als „intimate partner violence" bezeichnet, wobei sich letzterer Begriff spezifischer auf

V. Clemens und F. Köhler-Dauner teilen sich die Erstautorenschaft. die Gewalt zwischen Erwachsenen in einer Partnerschaft bezieht (u. a. Sigurdsson 2019). Die Prävalenzangaben hängen von der Definition, der Gesetzeslage im jeweiligen Land, aber auch von sozialen und ökonomischen Faktoren ab (Gulati und Kelly 2020). Je nach Untersuchung werden Häufigkeiten von 14-45\% aller Frauen genannt, die Gewalt in intimen Partnerschaften erleben (Thompson et al. 2006, Sigurdsson 2019). Gewalt in intimen Partnerschaften kommt sowohl gegen Frauen wie auch gegen Männer vor, wobei Frauen häufiger Opfer werden als Männer.

Diese Gewalterfahrungen können Auswirkungen auf die psychische Gesundheit haben (Howard et al. 2010). Insofern spielt Gewalt in intimen Partnerschaften auch eine Rolle im psychotherapeutischen Kontext, sowohl in der Behandlung von (Folge-)Störungen als auch ggf. als Raum, in dem Gewalterfahrungen erstmals thematisiert werden. Hierzu ist zu differenzieren, zwischen länger zurückliegenden Erfahrungen, auch Erfahrungen als Kind, und Gewalterfahrungen, die noch während einer Therapie stattfinden. Der psychotherapeutische Umgang ist dementsprechend unterschiedlich zu gestalten.

Eine Besonderheit ist, dass nicht allein das Erleben von Gewalt direkt gegen die eigene Person zu psychischen Folgen füh- ren kann, sondern auch das Beobachten, etwa als Kind, wenn es zu Gewalt zwischen den Eltern kommt. Beim Auftreten von Gewalt in intimen Partnerschaften ist oft das gesamte familiäre System beeinträchtigt. So ist z. B. das Risiko für eine Beeinträchtigung der Eltern-Kind-Interaktionen bei Müttern, die häuslicher Gewalt ausgesetzt sind, erhöht (Levendosky et al. 2011). Dies erscheint vor dem Hintergrund der Belastungen durch Angst und Gewalt nicht verwunderlich. Die Kinder selbst kommen häufig in einen Loyalitätskonflikt. Ein Loyalitätskonflikt tritt z. B. auf, wenn das Kind versucht, Zuneigung und gute Gefühle gegenüber jedem seiner Elternteile aufrechtzuerhalten, obwohl diese gewalttätig zueinander sind. Dies kann zu großen Belastungen führen. Wendet das Kind sich hingegen mehr einem Elternteil zu, kann es Schuldgefühle gegenüber dem anderen Elternteil bekommen. Die Schuldgefühle werden noch verstärkt, wenn die Elternteile jeweils die emotionale Zustimmung des Kindes erwarten oder sogar direkt einfordern.

Auch das Erleben anderer Kindheitsbelastungen, zu denen Gewalt in intimen Partnerschaften als eine Form, aber zudem auch noch weitere Formen von Haushaltsdysfunktionen und Kindesmisshandlung zählen (Felitti et al. 1998), erhöht das Risiko für potenziell schäd- 
liche Erziehungsmaßnahmen (Clemens et al. 2020). Insbesondere das Erleben mehrerer Arten von Kindheitsbelastungen ("adverse childhood experiences", ACE) steigert das Risiko für körperliche und psychische Probleme im Erwachsenenalter (Felitti et al. 1998; Clemens et al. 2018b; Riedl et al. 2020).

Metaanalysen zu diesem Thema zeigen, dass sowohl das Risiko für internalisierende Störungen, wie Depression, als auch für externalisierende Störungen, wie z. B. aggressive Verhaltensweisen im Rahmen einer Störung des Sozialverhaltens, wuchs, wenn in der Kindheit Gewalt zwischen den Eltern miterlebt wurde (Kitzmann et al. 2003; Wolfe et al. 2003). Die Risiken für soziale Probleme und schlechtere Schulergebnisse sind bei Kindern und Jugendlichen, die Gewalt zwischen den Eltern erleben, ebenfalls erhöht (Kitzmann et al. 2003). Ebenso konnte eine gesteigerte Bereitschaft für riskante Verhaltensweisen, einschließlich Drogenkonsum (Bair-Merritt et al. 2006) und Alkoholmissbrauch (Caetano et al. 2003), bei betroffenen Kindern/ Jugendlichen nachgewiesen werden.

Das Miterleben von Gewalt zwischen den Eltern ist häufig. In einer Untersuchung an einer repräsentativen Stichprobe der deutschen Bevölkerung gaben kürzlich knapp $10 \%$ der Befragten an, in der Kindheit Gewalt gegen die Mutter oder Stiefmutter miterlebt zu haben (Witt et al. 2019).

Durch die COVID-19-Pandemie hat sich das Leben von Familien in beispielloser Weise verändert (s. auch den Beitrag von Reis et al. in diesem Heft). Schätzungsweise $90 \%$ der Kinder und Jugendlichen waren zwischenzeitlich von Schulschließungen betroffen (UNESCO 2020). Soziale Kontakte außerhalb der eigenen Kernfamilie wurden stark eingeschränkt, viele Freizeitaktivitäten verboten (Team 2020). Eltern waren oft einer doppelten Belastung ausgesetzt: Sie mussten Kinder betreuen und ältere Kinder/Jugendliche beim Homeschooling unterstützen, während sie parallel dazu im Homeoffice arbeiteten. Der finanzielle Druck hat in vielen Familien aufgrund von Einkommenseinbußen zugenommen. Auch wirtschaftliche Probleme können zu Stress und konsekutiv zu Ehekonflikten und einer Zunahme häuslicher Gewalt führen (Elder 1974; Elder und Conger 2000; Stith et al. 2004). Berichte über häusliche Gewalt und Anrufe bei spezialisierten Beratungsangeboten haben während des ersten Lockdowns im Frühling 2020 in vielen Ländern, auch in Deutschland, zugenommen (UN women 2020).

Das Ziel der vorliegenden Studie war es daher, den Zusammenhang von Gewalt in intimen Partnerschaften und psychischen Problemen bei Kindern und Jugendlichen vor und während der COVID-19-Pandemie zu untersuchen. Auf Basis der dargestellten Literatur lauteten die Hypothesen, dass

- erhöhter wirtschaftlicher Druck, geringere Bildung und das Erleben von ACE Risikofaktoren für Gewalt in intimen Partnerschaften darstellen und

- Kinder aus Familien, in denen Gewalt zwischen den Eltern vorkommt, mehr psychische Probleme aufweisen, und dass diese Probleme während des Lockdowns stärker zugenommen haben als bei Kindern aus Familien ohne Gewalt zwischen den Eltern.

\section{Studiendesign und Unter- suchungsmethoden}

\section{Stichprobe und Prozedere}

Die Daten wurden über eine Online-Umfrage erhoben, die vom 18.05.2020 bis zum 21.07.2020 stattfand. Die Umfrage erfolgte über die Plattform unipark. Informationen über die Umfrage wurden über die Homepage der Uniklinik Ulm, soziale Medien und Printmedien sowie über bestehende Mailing-Listen von anderen Studien verbreitet.

Bevor Interessierte zu der eigentlichen Umfrage gelangten, wurden Informationen über die Studie und die Datenanalyse gegeben. Alle Teilnehmenden mussten vor der Teilnahme in eine elektronische Teilnahmeerklärung einwilligen. Die Teilnahme war anonym. Die Teilnehmenden konnten die Umfrage jederzeit ohne Angabe von Gründen beenden. Einschlusskriterien waren die Einwilligung in die Teilnahme und der Abschluss des 18. Lebensjahres. Insgesamt begannen 1826 Teilnehmende die Studie, 1405
(76,94\%) sendeten den Fragebogen zurück. In der vorliegenden Untersuchung wurden nur die Daten der Teilnehmenden ausgewertet, die den Fragebogen abschlossen und die angaben, minderjährige Kinder zu haben $(n=687)$.

Die Studie wurde in Übereinstimmung mit den ethischen Richtlinien durchgeführt, die in der Erklärung von Helsinki von 1964 festgelegt wurden. Nach Rücksprache mit der Ethikkommission der Universität Ulm bestand aufgrund des anonymen Charakters der Umfrage keine Notwendigkeit für ein Ethikvotum.

\section{Instrumente}

$\mathrm{Zu}$ den soziodemografischen Daten, die erhoben wurden, gehörten Alter, Geschlecht, Bildungsniveau, das subjektive Auskommen mit dem Haushaltseinkommen, Gewalt in der Beziehung und Einkommensveränderungen während der Pandemie. Belastende Kindheitserfahrungen der Erwachsenen wurden mithilfe der deutschen Version des Fragebogens „Adverse Childhood Experiences Questionnaire“ (ACE Questionnaire; Felitti et al. 1998) erfasst, eines Standardinstruments zur retrospektiven Erhebung belastender Kindheitserfahrungen mit zufriedenstellender interner Konsistenz (Cronbachs $\alpha=0,76$; Wingenfeld et al. 2011).

Die psychischen Probleme der Kinder wurden mithilfe einzelner Items aus der deutschen Elternversion des „Strengths and Difficulties Questionnaire" (SDQ) erhoben (Woerner et al. 2002), eines Screeninginstruments für Verhaltensauffälligkeiten bei Kindern und Jugendlichen (Goodman 1997). Um die Länge der Befragung zu begrenzen, erfolgte die Auswahl der Items selektiv. Folgende Items wurden genutzt: „unruhig, überaktiv, kann nicht lange stillsitzen“, „klagt häufig über Kopfschmerzen, Bauchschmerzen oder Übelkeit“, „ständig zappelig“, „oft unglücklich oder niedergeschlagen; weint häufig“, „hat viele Ängste; fürchtet sich leicht", "hat oft Wutanfälle; ist aufbrausend", "hat viele Sorgen; erscheint häufig bedrückt", „verhält sich gegenüber Erwachsenen oft widerwillig“ und „nervös oder anklam- 
Psychotherapeut 2021 -66:209-216 https://doi.org/10.1007/s00278-021-00501-w

(c) Der/die Autor(en) 2021

V. Clemens · F. Köhler-Dauner · F. Keller · U. Ziegenhain · J. M. Fegert · M. Kölch

\section{Gewalt in intimen Partnerschaften und psychische Probleme bei Kindern und Jugendlichen. Online- Survey während der COVID-19-Pandemie}

\section{Zusammenfassung}

Hintergrund. Die durch die "coronavirus disease 2019“ (COVID-19) ausgelöste Pandemie hat das Leben von Familien in beispielloser Weise verändert. Während des ersten Lockdowns wurden außerfamiliäre Kontakte erheblich reduziert. Viele Eltern mussten parallel ihre Kinder betreuen und von zu Hause aus arbeiten, während der ökonomische Druck zunahm.

Ziel der Arbeit (Fragestellung). Das Ziel der vorliegenden Studie war es, den Zusammenhang von Gewalt in intimen Partnerschaften und psychischen Problemen bei Kindern und Jugendlichen vor und während der COVID-19Pandemie zu untersuchen.

Material und Methoden. Mithilfe einer Online-Querschnittsumfrage wurden die Daten von 687 Eltern minderjähriger Kinder in Deutschland erhoben. Demografische und psychosoziale Prädiktoren für Gewalt in intimen Partnerschaften und Assoziationen mit psychischen Gesundheitsproblemen von Kindern vor, während und nach dem ersten Lockdown aufgrund der COVID-19Pandemie wurden analysiert. Die Umfrage fand vom 18.05.2020 bis zum 21.07.2020 statt. Der Lockdown in Deutschland begann am 23.03.2020 und endete über schrittweise Lockerungen - die ersten Schulen öffneten am 22.04.2020 wieder; die Öffnungen von Schulen, Kindergärten und Kitas zog sich bis Ende Juni 2020.

Ergebnisse. Ein geringeres Haushaltseinkommen und das Erleben von eigenen belastenden Kindheitserfahrungen erhöhen das Risiko, dass Studienteilnehmende Gewalt in ihrer Beziehung erfahren haben und darüber berichten. Kinder und Jugendliche, die in Familien leben, in denen Gewalt in intimen Partnerschaften vorkommt, wiesen nach Angaben der teilnehmenden Eltern vor und während der Pandemie häufiger höhere Werte für externalisierende Probleme auf, hinsichtlich emotionaler Probleme zeigten sich keine signifikanten Unterschiede. Diskussion. Belastende Kindheitserlebnisse erhöhen das Risiko für Gewalt in intimen Partnerschaften - und diese wiederum das Risiko für psychische Probleme der eigenen Kinder. Insofern sollte auch in der psychotherapeutischen Praxis systematisch nach entsprechenden Erfahrungen gefragt und entsprechende Behandlungsangebote sollte empfohlen werden.

\section{Schlüsselwörter}

CoVid-19-Pandemie - Intime Partnergewalt - Psychische Probleme - Kinder und Jugendliche · Belastende Kindheitserlebnisse (ACEs)

\section{Violence in intimate partnerships and mental problems in children and adolescents. Online survey during the COVID-19 pandemic}

\section{Abstract}

Background. The coronavirus disease 2019 (COVID-19) pandemic has changed the lives of families in an unprecedented way. During the first lockdown, extrafamilial contact was significantly reduced. Many parents had to look after their children and work from home while the economic pressure increased. Objective. The aim of the present study was to investigate the association between intimate partner violence (IPV) and mental health problems in children and adolescents before and during the COVID-19 pandemic.

Material and methods. In a cross-sectional online survey 687 parents of underaged children in Germany were included. Demographic and psychosocial predictors for IPV and associations with mental health problems of children before and during the first lockdown due to the COVID-19 pandemic were assessed. The survey ran from 18 May 2020 to 21 July 2020 and the lockdown in Germany began on 23 March 2020 and ended via a gradual relaxation. The first schools reopened on 22 April and the opening of schools, kindergartens and daycare centers extended to the end of June 2020.

Results. Lower household incomes and the experience of own adverse childhood experiences increased the risk of participants reporting violence in their relationship. According to the participating parents, children and adolescents living in families in which violence was reported by intimate partners were more likely to have higher levels of externalizing problems before and during the pandemic. There were no significant differences with respect to emotional problems.

Conclusion. Adverse childhood experiences increase the risk of intimate partner violence, which in turn increases the risk of mental health problems in the children. Therefore, in the psychotherapeutic practice questions should also be systematically asked about corresponding experiences and appropriate treatment offers should be recommended.

Keywords

CoVid-19 pandemic · Intimate partner violence $\cdot$ Mental health problems . Children and adolescents - Stressful childhood experiences (ACEs) mernd in neuen Situationen; verliert leicht das Selbstvertrauen“.

\section{Analyse der Daten}

Die statistischen Analysen wurden mit SPSS, Version 21, durchgeführt. Die Prädiktoren für das Auftreten häuslicher Ge- walt in der Beziehung wurden mithilfe einer logistischen Regression berechnet. Anhand von T-Tests wurden die psychischen Probleme von Kindern und Jugendlichen in Familien mit und ohne häusliche Gewalt jeweils vor und während der Pandemie verglichen.

\section{Ergebnisse}

\section{Stichprobe}

Die Teilnehmenden waren überwiegend Frauen $(n=614,89,5 \%)$. Das mittlere Alter der Teilnehmerinnen betrug 41,4 Jahre (Standardabweichung $[S D] \pm 7,4$ Jah- 


\begin{tabular}{|c|c|c|c|}
\hline & Frauen & Männer & $p$-Wert \\
\hline Anzahl der Teilnehmenden, $n$ (\%) & $615(89,5)$ & $72(10,5)$ & - \\
\hline Alter, $M( \pm S D ;$ Jahre) & $41,4( \pm 7,4)$ & $45,8( \pm 8,0)$ & $<0,001$ \\
\hline Alterspanne (Jahre) & $26-67$ & $33-71$ & - \\
\hline \multicolumn{4}{|l|}{ Höchster akademischer Abschluss, $n$ (\%) } \\
\hline Universitätsabschluss oder (Fach-) Abitur & $416(67,6)$ & $56(77,8)$ & - \\
\hline Anderer oder kein Schulabschluss & $199(32,4)$ & $16(22,2)$ & 0,079 \\
\hline Einkommensrückgang $>25 \%$ seit der Pandemie, & $69(11,2)$ & $6(8,3)$ & 0,455 \\
\hline \multicolumn{4}{|l|}{ Auskommen mit dem Haushaltseinkommen, $n(\%)$} \\
\hline Teilnehmende kommen gut aus & $384(62,4)$ & $44(61,1)$ & - \\
\hline Teilnehmende kommen aus & $163(26,3)$ & $21(29,2)$ & - \\
\hline Teilnehmende kommen gerade so aus & $51(8,3)$ & $7(9,7)$ & - \\
\hline Teilnehmende kommen kaum aus & $18(2,9)$ & $0(0,0)$ & 0,480 \\
\hline Gewalt in der Partnerschaft, $n(\%)$ & $34(6,1)$ & $2(2,9)$ & 0,413 \\
\hline $\begin{array}{l}\text { Anzahl belastender Kindheitserfahrungen, } M( \pm S D \text {; } \\
\text { Jahre) }\end{array}$ & $1,7( \pm 1,9)$ & $1,3( \pm 1,4)$ & 0,011 \\
\hline M Mittelwert, SD Standardabweichung & & & \\
\hline
\end{tabular}

re, Alterspanne 26 bis 67 Jahre), das der Teilnehmer 45,8 Jahre (SD $\pm 8,0$ Jahre, Altersspanne 33 bis 71 Jahre). Die Mehrheit der männlichen sowie weiblichen Teilnehmenden lebte mit einer ${ }^{*} \mathrm{~m}$ Partner ${ }^{*}$ in zusammen (w: $n=518,84,2 \% ; \mathrm{m}: n=65$, $90,3 \%)$. Der Bildungsgrad war hoch, die Mehrheit hatte als höchsten Abschluss das Abitur oder einen Fachhochschulbzw. Universitätsabschluss (w: $n=416$, $67,6 \%$; $: n=56,77,8 \%$ ). Eine Minderheit war finanziell so von der Pandemie betroffen, dass das Einkommen um mehr als ein Viertel reduziert war (w: $n=69$, $11,2 \%$; $\mathrm{m}: n=6,8,3 \%)$. Die demografischen Daten sind in • Tab. 1 zusammengefasst.

\section{Prädiktoren für häusliche Gewalt}

Teilnehmende, die mit ihrem Haushaltseinkommen nach eigener Einschätzung kaum auskamen, hatten ein signifikant höheres Risiko, häusliche Gewalt zu erfahren als Teilnehmende, die berichteten, mit ihrem Haushaltseinkommen auszukommen („odds ratio“ $[\mathrm{OR}]=9,58$, $p<0,01)$. Je mehr Formen belastender Kindheitserfahrungen von den Teilnehmenden in der Kindheit erlebt wurden, desto höher war ihr Risiko, von Gewalt in ihrer Beziehung betroffen $\mathrm{zu}$ sein $(\mathrm{OR}=1,21, p<0,05)$. Geschlecht, Lebensalter, Bildungsabschluss und Einbußen im Einkommen seit Beginn der
Pandemie stellten keine signifikanten Prädiktoren für häusliche Gewalt in der untersuchten Stichprobe dar (• Tab. 2).

\section{Psychische Probleme in}

Abhängigkeit von häuslicher Gewalt in der Familie

Im Vergleich zur Zeit vor der Pandemie nahmen alle problematischen Verhaltensweisen bei Kindern und Jugendlichen in Familien, in denen keine Gewalt unter den Eltern herrscht, nach Einschätzung der Eltern, signifikant zu (Unruhe: 1,36 vs. $1,53, p<0,001$, Schmerzen: 1,23 vs. $1,28, p=0,034$; zappelig: 1,25 vs. 1,38 , $p<0,001$; unglücklich/niedergeschlagen: 1,17 vs. $1,45, p<0,001$; Ängste: 1,26 vs. $1,39, p<0,001$; Wutanfälle: 1,46 vs. $1,73, p<0,001$; widerwillig: 1,45 vs. 1,66 , $p<0,001$; nervös/anklammernd: 1,31 vs. 1,43, $p<0,001)$. Bei den Kindern und Jugendlichen, in deren Familie Gewalt zwischen den Eltern herrschte, nahmen die problematischen Verhaltensweisen nach Einschätzung der Eltern ebenso zu, nicht signifikant war diese Zunahme lediglich bei den Items Schmerzen sowie nervös/anklammernd (Unruhe: 1,65 vs. $2,00, p=0,006$, Schmerzen: 1,23 vs. $1,35, p=0,103$; zappelig: 1,58 vs. 1,84 , $p=0,018$; unglücklich/niedergeschlagen: 1,23 vs. $1,65, p=0,001$; Ängste: 1,39 vs. $1,65, p=0,030$; Wutanfälle: 1,71 vs. 2,06 , $p=0,006$; widerwillig: 1,68 vs. 1,97 , $p=0,010$; nervös/anklammernd: 1,48 vs. $1,58, p=0,264)$. Kinder und Jugendliche, die in Familien leben, in denen von häuslicher Gewalt berichtet wurde, waren nach Einschätzung der Eltern signifikant häufiger zappelig, bereits vor der Pandemie $(\mathrm{t}(31,965)=-2,669, p<0,05)$ sowie während der Pandemie $(\mathrm{t}(555)=-3,870$, $p<0,001)$. Kinder und Jugendliche in Familien mit Partnerschaftsgewalt zeigten zudem signifikant häufiger Wutausbrüche, sowohl vor $(\mathrm{t}(551)=-2,302$, $p<0,05)$ als auch während der Pandemie $(\mathrm{t}(554)=-2,449, p<0,05)$. Ein signifikanter Unterschied in Abhängigkeit davon, ob zwischen den Eltern Gewalt berichtet wurde, fand sich des Weiteren dafür, ob Kinder/Jugendliche oft widerwillig gegenüber Erwachsenen sind. Der Mittelwert war sowohl vor $(\mathrm{t}(554)=-2,177$, $p<0,05)$ als auch während der Pandemie $(\mathrm{t}(554)=-2,345, p<0,05)$ signifikant höher als bei Kindern/Jugendlichen, aus deren Familien keine häusliche Gewalt berichtet wurde. Hinsichtlich emotionaler Probleme stellte sich folgender Trend dar: Emotionale Probleme sind bei Kindern und Jugendlichen, in deren Familien Gewalt zwischen den Eltern vorkommt, stärker ausgeprägt, und zudem werden die Unterschiede während der Pandemie eher größer. Eine statistische Signifikanz wurde hier jedoch nicht gefunden (• Abb. 1).

\section{Diskussion}

Inzwischen werden vermehrt Studienergebnisse zu möglichen psychischen Folgen als Auswirkung der COVID-19-Pandemie bzw. der entsprechenden Maßnahmen zur ihrer Eindämmung publiziert (u.a. Gulati und Kelly 2020; Lai et al. 2020; Torales et al. 2020; Vindegaard und Benros 2020). Hinsichtlich der häuslichen Situation gab es auch in Deutschland die Sorge, dass es in Familien, in denen das Risiko für das Erleben von Gewalt erhöht ist, ggf. zu einer Zunahme häuslicher Gewalt kommen könnte (Fegert et al. 2020). Generell können sowohl psychische Probleme bei Eltern als auch kindliche Psychopathologie ein Risikofaktor dafür sein, dass Vernachlässigung und Misshandlung vorkommen (Clemens et al. 2018a). Die vorliegende 


\begin{tabular}{|c|c|c|c|c|c|c|}
\hline & $x^{2}(d f)$ & $\begin{array}{l}p \text { - } \\
\text { Wert }\end{array}$ & $\mathbf{R}^{2}$ & $\begin{array}{l}\text { Odds } \\
\text { ratio }\end{array}$ & $95 \%-K I$ & $\begin{array}{l}p \text { - } \\
\text { Wert }\end{array}$ \\
\hline Modell 1 & $1,328(2)$ & 0,515 & 0,006 & - & - & - \\
\hline Männliches Geschlecht & - & - & - & 0,473 & $0,109-2,049$ & 0,317 \\
\hline Alter (Jahre) & - & - & - & 0,997 & $0,950-1,047$ & 0,905 \\
\hline Modell 2 & $3,298(3)$ & 0,348 & 0,015 & - & - & - \\
\hline Männliches Geschlecht & - & - & - & 0,497 & $0,115-2,156$ & 0,350 \\
\hline Alter (Jahre) & - & - & - & 0,997 & $0,950-1,046$ & 0,899 \\
\hline $\begin{array}{l}\text { Universitätsabschluss oder } \\
\text { (Fach-)Abitur }\end{array}$ & - & - & - & 0,604 & $0,302-1,209$ & 0,154 \\
\hline Modell 3 & $\begin{array}{l}11,956 \\
(5)\end{array}$ & 0,035 & 0,054 & - & - & - \\
\hline Männliches Geschlecht & - & - & - & 0,535 & $0,122-2,337$ & 0,405 \\
\hline Alter (Jahre) & - & - & - & 1,008 & 0,959-1,059 & 0,755 \\
\hline $\begin{array}{l}\text { Universitätsabschluss oder } \\
\text { (Fach-)Abitur }\end{array}$ & - & - & - & 0,545 & $0,268-1,109$ & 0,094 \\
\hline $\begin{array}{l}\text { Einkommenssenkung um } \\
>25 \%\end{array}$ & - & - & - & 1,772 & $0,484-6,489$ & 0,388 \\
\hline $\begin{array}{l}\text { Teilnehmende kommen kaum } \\
\text { mit dem Haushalteinkommen } \\
\text { aus }\end{array}$ & - & - & - & 11,823 & $2,457-56,901$ & 0,002 \\
\hline Modell 4 & $\begin{array}{l}16,266 \\
(6)\end{array}$ & 0,012 & 0,074 & - & - & - \\
\hline Männliches Geschlecht & - & - & - & 0,597 & $0,136-2,624$ & 0,495 \\
\hline Alter (Jahre) & - & - & - & 1,004 & $0,955-1,056$ & 0,866 \\
\hline $\begin{array}{l}\text { Universitätsabschluss oder } \\
\text { (Fach-)Abitur }\end{array}$ & - & - & - & 0,608 & $0,295-1,252$ & 0,177 \\
\hline $\begin{array}{l}\text { Einkommenssenkung um } \\
>25 \%\end{array}$ & - & - & - & 1,935 & $0,521-7,191$ & 0,324 \\
\hline $\begin{array}{l}\text { Teilnehmende kommen kaum } \\
\text { mit dem Haushalteinkommen } \\
\text { aus }\end{array}$ & - & - & - & 9,584 & $1,957-46,940$ & 0,005 \\
\hline ACE-Summenscore & - & - & - & 1,205 & $1,017-1,427$ & 0,031 \\
\hline \multicolumn{7}{|c|}{ 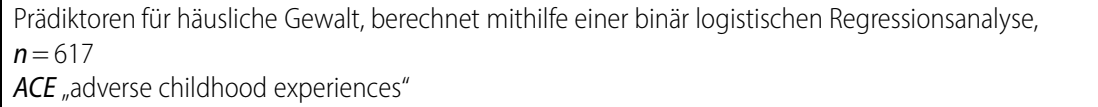 } \\
\hline
\end{tabular}

Untersuchung ist gemäß dem Wissen eine der ersten, die unmittelbar während der Lockdown-Maßnahmen in Deutschland Eltern im Zusammenhang mit Vorerfahrung eigener ACE und dem kindlichen problematischen Verhalten befragt hat.

Im Laufe der Untersuchung nahmen die psychischen Probleme bei Kindern und Jugendlichen zu. Diese Erkenntnis stimmt mit anderen Ergebnissen überein (Ravens-Sieberer et al. 2020) und weist auf die Belastung von Kindern und Jugendlichen durch die Pandemie sowie durch die dadurch bedingten Maßnahmen, wie Schulschließungen, Wegfall von außerfamiliären Kontakten, hin. Generell ist aber festzuhalten, in der Familie festgestellt wurden. Dies mag daran liegen, dass internalisierende Störungen generell von Eltern weniger gut wahrgenommen werden als externalisierende Störungen, insbesondere wenn Eltern selbst belastet sind (Los Reyes und Kazdin 2005; Kelley et al. 2017). Der Befund verdeutlicht umso mehr, dass spezielle Gruppen, die bereits vor der Pandemie besonderen Risiken ausgesetzt waren, dies auch während der Pandemie sind.

Eine Untersuchung zur Rolle von Gewalt in intimen Partnerschaften zeigte, dass diese und psychische Misshandlung sich auf psychische Langzeitfolgen, wie Depressionen und Angstzustände in Kombination, stärker auswirken als sexueller Missbrauch durch Familienmitglieder oder Bekannte (Teicher et al. 2006). Es scheint also, dass nicht nur „klassische“ Misshandlungsformen wie z.B. sexueller Missbrauch, sondern auch Exposition gegenüber häuslicher und psychischer Gewalt - Misshandlungsformen, die häufig als weniger „schlimm“ erachtet werden - die kindliche Entwicklung maßgeblich schädigen können (Clemens et al. 2018b, 2020). Insofern weisen die erhobenen Daten darauf hin, dass es vermutlich einer stärkeren adjustierten Risikoeinschätzung dazu bedarf, welche Personen ggf. auch unter Pandemiebedingungen der Unterstützung bedürfen. Kinder und Jugendliche, die in Haushalten mit Gewalt zwischen den Eltern aufwachsen, würden hierzu gehören.

Zudem legen Studienergebnisse nahe, dass ein Zusammenhang existiert, zwischen in der Kindheit miterlebter Gewalt unter den Eltern und dem Risiko, dass Söhne im Erwachsenenalter selbst Täter sowie Töchter Opfer häuslicher Gewalt werden (Wood und Sommers 2011). Diese Ergebnisse weisen auf eine intergenerationelle Übertragung von Gewalt in intimen Partnerschaften hin und verdeutlichen die Notwendigkeit, Kinder zu schützen, um diesen „Teufelskreis der Gewalt" $\mathrm{zu}$ unterbrechen.

Ein weiterer Risikofaktor für das Auftreten von Gewalt in intimen Partnerschaften bestand, wenn das Haushaltseinkommen kaum ausreichte. Dieses Ergebnis stimmt mit anderen Unter- 


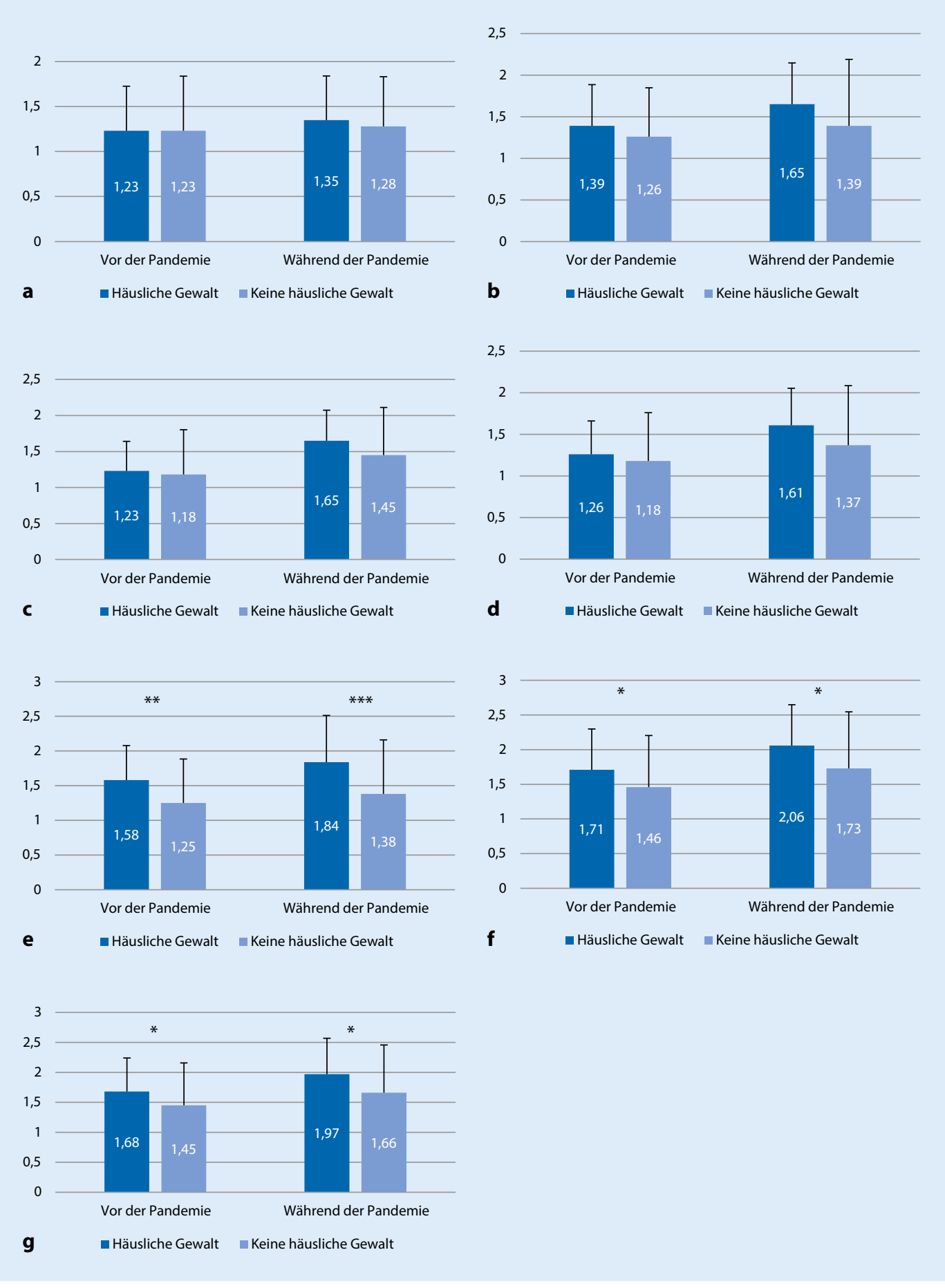

Abb. $1 \varangle$ Psychische Probleme bei Kindern und Jugendlichen in Abhängigkeit vom Auftreten von Gewalt zwischen den Eltern. a Häufiges Klagen über Kopfschmerzen, Bauchschmerzen oder Übelkeit; b viele Ängste, fürchtet sich leicht; c oft unglücklich, niedergeschlagen, weint oft; $d$ viele Sorgen, häufig bedrückt; e ständig zappelig; $f$ oft Wutanfälle, aufbrausend; $g$ oft widerwillig gegenüber Erwachsenen $\left(n=554-558 .{ }^{*} p<0,05\right.$, $\left.{ }^{* *} p<0,01,{ }^{* * *} p<0,001\right)$

suchungen überein, die zeigen, dass wirtschaftliche Probleme mit dem Auftreten häuslicher Gewalt assoziiert sind (Elder 1974; Elder und Conger 2000; Stith et al. 2004). Inzwischen liegen auch diesbezügliche Publikationen im Zusammenhang mit der COVID-Pandemie vor (Sharma und Borah 2020; Bryant et al. 2020). Auch eine Reduk- tion des Einkommens war mit einem erhöhten Risiko für Partnerschaftsgewalt verbunden. Hier muss als Limitation der Studie benannt werden, dass die Stichprobe ein sehr hohes Bildungsniveau aufwies und zudem die Einschätzung des Haushaltseinkommens rein subjektiv erfolgte. Interessanterweise scheint es jedoch v.a. die subjektive Erwartung der wirtschaftlichen Bedrohung sein, die das Risiko für intrafamiliäre Gewalt während Rezessionen steigert (Lee et al. 2013; Schneider et al. 2017). Allerdings ist hervorzuheben, dass nur $7 \%$ der Varianz durch dieses Modell erklärt werden können. Es ist es also anzunehmen, dass noch weitere wichtige Risikofaktoren, die im Rahmen der Umfrage nicht erho- 
ben wurden, eine Rolle spielen. Aus der Literatur bekannte Faktoren sind u.a. psychische Erkrankungen und Substanzabusus beim Partner/bei der Partnerin, kultureller Hintergrund, soziale Unterstützung und Stress (Capaldi et al. 2012).

\section{Limitationen der Studie}

Die Ergebnisse der vorliegenden Untersuchung unterstreichen bereits bekannte Befunde aus der Wissenschaft auch für diese deutsche Stichprobe vor und während des ersten Lockdowns. Dennoch sind einige Limitationen hervorzuheben. Die Ergebnisse beruhen nicht auf der Umfrage einer repräsentativen Stichprobe. Die Angaben zu psychischen Problemen bei Kindern und Jugendlichen basieren auf Antworten der Eltern. Hierbei können insbesondere internalisierende Probleme unterschätzt worden sein. Zudem handelt es sich um eine Querschnittserhebung. Die retrospektive Erfassung von psychischen Problemen, aber auch Kindheitsbelastungen kann zu Verzerrungen führen. Auch weitere familiäre Faktoren, wie z. B. Zahl, Geschlecht und Alter der Kinder, könnten Einfluss auf die untersuchten Zusammenhänge haben und zu Verzerrungen führen. Da Gewalt in intimen Partnerschaften häufig mit anderen Formen von Kindesmisshandlungen einhergeht (Clemens et al. 2019), ist es wahrscheinlich, dass die betroffenen Kinder noch mehr Formen von ACE erlebt haben - was ebenso zu Verzerrungen führen kann.

\section{Fazit für die Praxis}

- Die gezeigten Daten legen nahe, dass Gewalt in intimen Partnerschaften das Risiko für psychische Probleme erhöht - auch transgenerational. Kinder, die im häuslichen Kontext Gewalt miterleben, entwickeln mehr psychische Auffälligkeiten, als Kinder, die diese Erfahrungen nicht machen.

- In der psychotherapeutischen Praxis sollte systematisch nach entsprechenden Erfahrungen gefragt und ggf. sollten etwaige psychische Probleme der Kinder erhoben werden.
- Da externalisierendes Verhalten von Kindern wiederum im Zusammenhang mit dem ungünstigen Erziehungsverhalten von Eltern steht, kann dieses die Gefahr für häusliche Gewalt auch gegen Kinder erhöhen. - Bei Hinweisen auf das Vorkommen von häuslicher Gewalt und kindlichen psychischen Auffälligkeiten sind entsprechende Behandlungsangebote auch für das Kind zu empfehlen.

\section{Korrespondenzadresse}

\section{Dr. Vera Clemens}

Klinik für Kinder- und Jugendpsychiatrie/ Psychotherapie, Universitätsklinikum Ulm Steinhövelstr. 5, 89073 Ulm, Deutschland vera.clemens@uni-ulm.de

Funding. Open Access funding enabled and organized by Projekt DEAL.

\section{Einhaltung ethischer Richtlinien}

Interessenkonflikt. J.M. Fegert hat Forschungsförderungen von der EU, DFG (Deutsche Forschungsgemeinschaft), BMG (Bundesministerium für Gesundheit), BMBF (Bundesministerium für Bildung und Forschung), BMFSFJ (Bundesministerium für Familie, Senioren, Frauen und Jugend), G-BA Innovationsfonds, mehreren Landesministerien, der Landesstiftung Baden-Württemberg, Volkswagen-Stiftung, PorticusStiftung, des Bistum Rottenburg-Stuttgart erhalten. Darüber hinaus erhielt er in den letzten 5 Jahren von APK, Deutschlandfunk, DFG, DJI, DKSB, Infectopharm, med update, UNICEF, mehreren Universitäten, Berufsverbänden, politischen Stiftungen sowie Bundesund Landesministerien Reisestipendien, Honorare und Sponsoring für Kongresse und medizinische Fortbildung. Professor Fegert hält keine Aktien von Pharmaunternehmen. M. Kölch hat Forschungsförderung vom BMBF (Bundesministerium für Bildung und Forschung), von der BzGA (Bundeszentrale für gesundheitliche Aufklärung), vom UBSKM (Unabhängiger Beauftragter gegen sexuellen Kindesmissbrauch), Land Mecklenburg-Vorpommern, G-BA Innovationsfonds erhalten. Er hat Janssen als wissenschaftlicher Experte beraten. Kein Interessenkonflikt im Zusammenhang mit der Publikation. V. Clemens, F. KöhlerDauner, F. Keller und U. Ziegenhain geben an, dass kein Interessenkonflikt besteht.

Alle beschriebenen Untersuchungen am Menschen oder an menschlichem Gewebe wurden mit Zustimmung der zuständigen Ethikkommission, im Einklang mit nationalem Recht sowie gemäß der Deklaration von Helsinki von 1975 (in der aktuellen, überarbeiteten Fassung) durchgeführt. Alle Studienteilnehmenden haben vor Beginn der Teilnahme ihr Einverständnis erteilt.

Open Access. Dieser Artikel wird unter der Creative Commons Namensnennung 4.0 International Lizenz veröffentlicht, welche die Nutzung, Vervielfältigung,
Bearbeitung, Verbreitung und Wiedergabe in jeglichem Medium und Format erlaubt, sofern Sie den/die ursprünglichen Autor(en) und die Quelle ordnungsgemäß nennen, einen Link zur Creative Commons Lizenz beifügen und angeben, ob Änderungen vorgenommen wurden.

Die in diesem Artikel enthaltenen Bilder und sonstiges Drittmaterial unterliegen ebenfalls der genannten Creative Commons Lizenz, sofern sich aus der Abbildungslegende nichts anderes ergibt. Sofern das betreffende Material nicht unter der genannten Creative Commons Lizenz steht und die betreffende Handlung nicht nach gesetzlichen Vorschriften erlaubt ist, ist für die oben aufgeführten Weiterverwendungen des Materials die Einwilligung des jeweiligen Rechteinhabers einzuholen.

Weitere Details zur Lizenz entnehmen Sie bitte der Lizenzinformation auf http://creativecommons.org/ licenses/by/4.0/deed.de.

\section{Literatur}

Bair-Merritt MH, Blackstone M, Feudtner C (2006) Physical health outcomes of childhood exposure to intimate partnerviolence: a systematic review. Pediatrics 117(2):e278-290

Bryant DJ, Oo M, Damian AJ (2020) The rise of adverse childhood experiences during the COVID-19 pandemic. Psychol Trauma 12(S1):S193-S194. https://doi.org/10.1037/tra0000711

Caetano R, Field CA, Nelson S (2003) Association between childhood physical abuse, exposure to parental violence, and alcohol problems in adulthood. J Interpers Violence 18(3):240-257

Capaldie DM, Knoble NB, Shortt JW, Kim HK (2012) A systematic review of risk factors for intimate partner violence. Partn Abus 3(2):231-280

Clemens V, Berthold O, Fegert JM, Kölch M (2018a) Kinder psychisch erkrankter Eltern. Nervenarzt 89(11):1262-1270

Clemens V, Huber-Lang M, Plener PL, Brahler E, Brown RC, Fegert JM (2018b) Association of child maltreatment subtypes and long-term physical health in a German representative sample. Eur J Psychotraumatol 9(1):1510278

Clemens V, Berthold O, Witt A, Sachser C, Brähler E, Plener PL, Strauß B, Fegert JM (2019) Child maltreatment is mediating long-term consequences of household dysfunction in a population representative sample. Eur Psychiatry 58:10-18

Clemens V, Berthold O, Witt A, Brähler E, Plener PL, Fegert JM (2020) Childhood adversities and later attitudes towards harmful parenting behaviour including shaking in a German populationbased sample. Child Abus Rev 29(3):269-281

Elder GHJ (1974) Children of the Great Depression: social changes in life experience. Westview, Boulder

Elder GHJ, Conger RD (2000) Children of the land: adversity and success in rural America. University of Chicago Press, Chicago

Fegert JM, Berthold O, Clemens V, Kölch M (2020) COVID-19-Pandemie: Kinderschutz ist systemrelevant. Dtsch Arztebl Int 117(14):A-703

Felitti VJ, Anda RF, Nordenberg D, Williamson DF, Spitz AM, Edwards V, Koss MP, Marks JS (1998) Relationship of childhood abuse and household dysfunction to many of the leading causes of death in adults: the adverse childhood experiences (ACE) study. Am J Prev Med 14(4):245-258 
Goodman R (1997) The strengths and difficulties questionnaire: a research note. J Child Psychol Psychiatry 38(5):581-586

Gulati G, Kelly BD (2020) Domestic violence against women and the COVID-19 pandemic: What is the role of psychiatry? Int JLaw Psychiatry 71:101594

Howard LM, Trevillion K, Agnew-Davies R (2010) Domestic violence and mental health. Int Rev Psychiatry 22(5):525-534

Imperial College COVID-19 Response Team (2020) Estimating the number of infections and the impact of non-pharmaceutical interventions on COVID-19 in 11 European countries. https:// www.imperial.ac.uk/media/imperial-college/ medicine/sph/ide/gida-fellowships/ImperialCollege-COVID19-Europe-estimates-and-NPIimpact-30-03-2020.pdf. Zugegriffen: 11. März 2021

KelleyML, Bravo AJ, HamrickHC, Braitman AL, White TD Jenkins J (2017) Parents' reports of children's internalizing symptoms: associations with parents' mental health symptoms and substance use disorder. J Child Fam Stud 26(6):1646-1654. https://doi.org/10.1007/s10826-017-0677-9

Kitzmann KM, Gaylord NK, Holt AR, Kenny ED (2003) Child witnesses to domestic violence: a meta-analytic review. J Consult Clin Psychol 71(2):339-352

Lai J, Ma S, Wang Y, Cai Z, Hu J, Wei N, Wu J, Du H, Chen T, Li R, Tan H, Kang L, Yao L, Huang M, Wang $\mathrm{H}_{\text {, }}$ Wang G, Liu Z, Hu S (2020) Factors associated with mental health outcomes among health care workers exposed to coronavirus disease 2019 JAMA Netw Open 3(3):e203976

Lee D, Brooks-Gunn J, McLanahan SS, Notterman D, Garfinkel I (2013) The Great Recession, genetic sensitivity, and maternal harsh parenting. Proc Natl Acad SciU S A 110(34):13780-13784

Levendosky A, Bogat GA, Huth-Bocks A (2011) The influence of domestic violence on the development of the attachment relationship between mother and young child. Psychoanal Psychol.https://doi.org/10.1037/a0024561

Los Reyes A de, Kazdin AE (2005) Informant discrepancies in the assessment of childhood psychopathology: a critical review, theoretical framework, and recommendations for further study. Psychol Bull 131(4):483-509. https://doi. org/10.1037/0033-2909.131.4.483

Ravens-Sieberer U, Kaman A, Otto C, Adedeji A, Devine J, Erhart M, Napp A-K, Becker M, BlanckStellmacher U, Löffler C, Schlack R, Hurrelmann K (2020) Psychische Gesundheit und Lebensqualität von Kindern und Jugendlichen während der COVID-19-Pandemie - Ergebnisse der COPSYStudie. Dtsch Arztebl Int 117(48):828-829

Riedl D, Lampe A, Exenberger S, Nolte T, Trawöger I, Beck T (2020) Prevalence of adverse childhood experiences (ACEs) and associated physical and mental health problems amongst hospital patients: results from a cross-sectional study. Gen Hosp Psychiatry 64:80-86

Schneider W, Waldfogel J, Brooks-Gunn J (2017) The Great Recession and risk for child abuse and neglect. Child Youth Serv Rev 72:71-81

Sharma A, Borah SB (2020) Covid-19 and domestic violence: an indirect path to social and economic crisis. J Fam Viol. https://doi.org/10.1007/ s10896-020-00188-8

Sigurdsson EL (2019) Domestic violence - are we up to the task? Scand J Prim Health Care 37(2):143-144

Stith SM, Smith DB, Penn CE, Ward DB, Tritt D (2004) Intimate partner physical abuse perpetration and victimization risk factors: a meta-analytic review. Aggress Violent Behav 10(1):65-98

Teicher MH, Samson JA, Polcari A, McGreenery CE (2006) Sticks, stones, and hurtful words: relative effects of various forms of childhood maltreatment. Am J Psychiatry 163(6):993-1000

Thompson RS, Bonomi AE, Anderson M, Reid RJ, Dimer JA, Carrell D, Rivara FP (2006) Intimate partner violence: prevalence, types, and chronicity in adult women. Am J Prev Med 30(6):447-457

Torales J, O'Higgins M, Castaldelli-Maia JM, Ventriglio A (2020) The outbreak of COVID-19 coronavirus and its impact on global mental health. Int J Soc Psychiatry66(4):317-320

UN women (2020) COVID-19 and ending violence against women and girls. https:// www.unwomen.org/-/media/headquarters/ attachments/sections/library/publications/ 2020/issue-brief-covid-19-and-endingviolence-against-women-and-girls-en.pdf? la=en\&vs=5006. Zugegriffen: 11. März 2021

UNESCO (2020) COVID-19 educational disruption and response. https://en.unesco.org/covid19/ educationresponse.Zugegriffen: 11. März 2021

Vindegaard N, Benros ME (2020) COVID-19 pandemic and mental health consequences: systematic review of the current evidence. Brain Behav Immun 89:531-542

Wingenfeld K, Schäfer I, Terfehr K, Grabski H, Driessen M, Grabe H, Löwe B, Spitzer C (2011) Reliable, valide und ökonomische Erfassung früher Traumatisierung: Erste psychometrische Charakterisierung der deutschen Version des Adverse Childhood Experiences Questionnaire (ACE). Psychother Psychosom Med Psychol 61(01):e10-e14

Witt A, Sachser C, Plener PL, Brähler E, Fegert JM (2019) The prevalence and consequences of adverse childhood experiences in the German population. Dtsch Arztebl Int 116(38):635-642

Woerner W, Becker A, Friedrich C, Rothenberger A, Klasen H, Goodman R (2002) Normierung und Evaluation der deutschen Elternversion des Strengths and Difficulties Questionnaire (SDQ) Ergebnisse einer repräsentativen Felderhebung. Z Kinder Jugendpsychiatr Psychother 30(2):105-112

Wolfe DA, Crooks CV, Lee V, McIntyre-Smith A, Jaffe PG (2003) The effects of children's exposure to domestic violence: a meta-analysis and critique. Clin Child Fam Psychol Rev 6(3):171-187

Wood SL, Sommers MS (2011) Consequences of intimate partner violence on child witnesses: a systematic review of the literature. J Child Adolesc Psychiatr Nurs 24(4):223-236
Hier steht eine Anzeige. Springer 